Nutrition Science en évolution

La revue de l'Ordre professionnel des diététistes du Québec

\title{
Honoraires et avance de paiement
}

\section{Janick Perreault}

Volume 16, numéro 1, printemps 2018

URI : https://id.erudit.org/iderudit/1048944ar

DOI : https://doi.org/10.7202/1048944ar

Aller au sommaire du numéro

Éditeur(s)

Ordre professionnel des diététistes du Québec

ISSN

2561-620X (numérique)

Découvrir la revue

Citer cet article

Perreault, J. (2018). Honoraires et avance de paiement. Nutrition Science en évolution, 16(1), 29-30. https://doi.org/10.7202/1048944ar d'utilisation que vous pouvez consulter en ligne.

https://apropos.erudit.org/fr/usagers/politique-dutilisation/ 


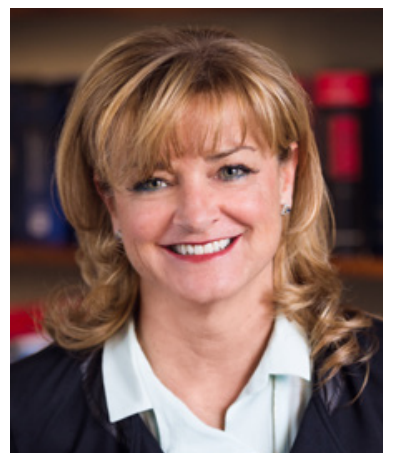

HONORAIRES ET AVANCE DE PAIEMENT

Il existe un dicton qui dit que toute peine mérite salaire, signifiant qu'il est juste d'obtenir une rétribution peu importe la nature du travail que l'on exécute. Or, si tout travail mérite rémunération, il en va ainsi de tout service professionnel. Et dans cet objectif, l'idée de demander une avance de paiement est intéressante. Mais, les diététistes/nutritionnistes peuvent-ils recevoir des paiements à l'avance? C'est à cette question qu'est destinée la présente chronique.

Maître Janick Perreault, Ad. E., Dt.P., LL.B., LL.M.*

\section{Communiqué de L'OPDQ concernant les avances d'honoraires}

Le 13 mars 2018, l'Ordre professionnel des diététistes du Québec (OPDQ) envoyait un communiqué à ses membres. Il annonçait une nouvelle directive: les avances de paiement par les clients. Si cette directive semble viser les services des diététistes/nutritionnistes en pratique privée ou à contrat, elle s'applique en fait à tout type de services nutritionnels.

Ce communiqué faisait suite à un récent avis important de l'Office des professions concernant les avances d'honoraires des professionnels. Une avance signifie tout paiement, en partie ou en totalité, du montant des honoraires ou des frais qu'un diététiste/ nutritionniste pourrait engager avant de rendre ses services professionnels.

Les codes de déontologie de plusieurs ordres professionnels, dont celui des diététistes/nutritionnistes, permettent les avances d'honoraires ou de débours, sans les encadrer par règlement. L'Office des professions demande désormais aux ordres concernés de se conformer au Code des professions $^{[1]}$. Ce code prévoit que les membres d'un ordre professionnel ne peuvent, dans l'exercice de leur profes- sion, détenir pour le compte d'un client ou d'une autre personne des sommes ou des biens, notamment des avances d'honoraires, que si le conseil d'administration l'autorise expressément par règlement ${ }^{[2]}$. Un tel règlement doit déterminer les modalités et les normes relatives : 1) à la détention et à la disposition des sommes d'argent; 2) à la tenue et à l'inspection des livres et registres des membres; 3 ) à la tenue et à l'inspection d'un compte en fidéicommis, s'il y a lieu ${ }^{[3]}$.

Lorsqu'un tel règlement existe, le conseil d'administration doit prévoir l'indemnisation d'un réclamant à la suite de l'utilisation par un membre de sommes ou de biens à des fins autres que celles prévues dans le cadre de l'exercice de sa profession. Le conseil d'administration doit, par règlement, déterminer la procédure d'indemnisation; s'il y a lieu, les modalités d'établissement d'un fonds d'indemnisation et les règles d'administration et de placement des montants seront aussi précisées ${ }^{[4]}$.

L'OPDQ a déjà amorcé des travaux afin d'évaluer s'il y a lieu d'adopter un tel règlement pour mieux encadrer la pratique des diététistes/nutritionnistes et se conformer à la directive de l'Office des professions.

\section{Code de déontologie des diététistes/nutritionnistes}

L'actuel Code de déontologie des diététistes édicte que «le diététiste ne doit pas exiger d'avance le paiement complet de ses services ${ }^{[5]}$, laissant ainsi la possibilité de demander une avance partielle. Rappelons toutefois que la détention pour le compte d'un client des avances d'honoraires n'est possible que si le Conseil d'administration l'autorise expressément par règlement. Or, un tel règlement n'existe pas pour les diététistes/nutritionnistes.

Ainsi, malgré l'actuel libellé du code de déontologie permettant aux diététistes d'exiger un paiement partiel de leurs services, l'OPDQ se devait de rappeler à ses membres qu'ils ne sont pas autorisés à réclamer des avances d'honoraires (incluant un paiement partiel perçu d'avance), et ce, tant que le conseil d'administration n'a pas évalué pleinement le risque et n'a pas déterminé la meilleure façon d'encadrer la pratique de ses membres.

L'OPDQ doit évaluer la nécessité d'adopter un tel règlement et sa pertinence considérant la réalité de ses membres. L'Ordre doit analyser les risques financiers au cas où il devrait rembourser un client en cas de vol 
30 ou de fraude par exemple. Un tel règlement encadrerait les modalités de détention des sommes par les professionnels et déterminerait les modalités d'indemnisation par l'Ordre d'un client dont les sommes auraient, par exemple, disparu du compte d'un professionnel.

\section{La fixation et le paiement d'honoraires}

Le code de déontologie encadre la fixation et le paiement des honoraires. Le diététiste/nutritionniste doit demander des honoraires justes, raisonnables et proportionnels aux services rendus. Il doit notamment tenir compte des facteurs suivants dans la fixation de ses honoraires : 1) le temps consacré à l'exécution du service professionnel;2) la difficulté et l'importance du service; et 3) la prestation de services inhabituels ou exigeant une compétence ou une célérité exceptionnelle ${ }^{[6]}$. Le diététiste/nutritionniste doit informer à l'avance son client du coût approximatif de ses services et des modalités de paiement. Également, il doit lui fournir toutes les explications nécessaires à la compréhension de son relevé d'honoraires ${ }^{[7]}$.

Il est difficile de parler des avances de paiement sans mentionner les forfaits. D'ailleurs, certains membres se sont questionnés à savoir s'ils pouvaient encore offrir des forfaits. Or, même avant cette nouvelle directive, les forfaits soulevaient une série de questionnements. Ainsi, en ne pouvant pas exiger à l'avance le paiement complet des services, il est difficile d'exiger le paiement complet d'un forfait avant même que tous les services qu'il englobe n'aient été rendus. Ceci est d'autant plus vrai que le diététiste/ nutritionniste doit éviter de poser ou de multiplier sans raison suffisante des actes professionnels dans l'exercice de sa profession et s'abstenir de poser un acte inapproprié ou disproportionné au besoin de son client ${ }^{[8]}$. Ce faisant, il est difficile de «forcer le respect» de ce qui a été prévu dans un forfait.
Si au cours des suivis, les actes professionnels ne sont plus requis, il faut y mettre fin; le coût du forfait peut devoir être ajusté, et ce, pour respecter l'obligation de charger des honoraires justes, raisonnables et proportionnels aux services rendus. Nous ne nous prononçons pas ici sur le principe de forfait puisque ce sujet fait partie des discussions du groupe de travail sur la révision du Code de déontologie des diététistes.

Il est également difficile de parler des avances de paiement sans parler du travail au sein d'une société qui perçoit le paiement des honoraires avant d'en redistribuer le paiement au diététiste/nutritionniste. Dans de tels cas, le professionnel doit s'assurer que la société, à titre de tiers qui prélève les honoraires, ne contrevient pas au code de déontologie. Par conséquent, des honoraires ne peuvent pas être perçus pour le diététiste/nutritionniste avant que le service ne soit effectivement rendu. En d'autres mots, le coût d'une consultation doit être acquitté uniquement lors de la consultation et non pas à l'avance.

Il est aussi difficile de parler des avances de paiement sans évoquer le souci d'être payé pour les services rendus. Dans la mesure où les avances d'honoraires ne sont pas autorisées, comment faire pour s'assurer que le patient ou le client paiera effectivement après avoir reçu le service? À cet égard, il faut savoir que les obligations professionnelles existent nonobstant la question du paiement des honoraires. Néanmoins, le diététiste/nutritionniste peut refuser ou cesser d'agir pour le compte d'un client ${ }^{[9]}$ en cas de refus de paiement des honoraires, car il s'agit d'un motif juste et raisonnable. Il faut aussi savoir que pour les comptes en souffrance, le diététiste/nutritionniste peut percevoir des intérêts, mais seulement après en avoir dûment avisé son client et que le taux d'intérêt exigé est raisonnable ${ }^{[10]}$. Toute politique de paiement des honoraires devrait être accessible aux clients.
Enfin, il est possible de percevoir des honoraires en cas de non-respect des rendez-vous si le client en a été avisé au préalable et que le montant est juste, raisonnable et proportionnel aux services ${ }^{[11]}$. En effet, ce paiement vise à compenser le temps perdu qui aurait pu être consacré à l'exécution d'un service professionnel.

Même si les avances de paiement sont interdites, soulignons que cela n'empêche pas de demander à l'avance un numéro de carte de crédit et de l'utiliser lors de la consultation. Cette façon de faire s'avère particulièrement utile pour les services en ligne.

\section{Conclusion}

Jusqu’à nouvel ordre, les diététistes/ nutritionnistes ne peuvent pas demander des avances de paiement, et ce, même s'il s'agit d'une avance partielle, alors que leur actuel code de déontologie ne les empêche qu'à l'égard d'une avance pour le paiement complet ${ }^{[12]}$. La directive s'applique à tout type de services nutritionnels, qu'il s'agisse de cours de groupe, de services en ligne, etc. Tous les membres de l'OPDQ doivent donc s'y conformer.

Finalement, notons que l'OPDQ amorce des travaux de réflexion sur les différentes méthodes de facturation et compte consulter des membres en pratique privée.

N.D.L.R. * L'auteure est diététiste/ nutritionniste, avocate et présidente du comité sur la pratique illégale de l'Ordre professionnel des diététistes du Québec.

\footnotetext{
Références

1. Code des professions, RLRQ, chapitre C-26.

2. Code des professions, RLRQ, chapitre C-26, article 89.

3. Id.

4. Id., article 89.1.

5. Code de déontologie des diététistes, RLRQ, chapitre C-26, r. 97, art. 33.

6. Id., art. 31 .

7. Id., art. 32 .

8. Id., art. 13.

9. Id., art. 16.

10. Id., art. 34

11. Id., art. 31.

12. Id., art. 33.
} 